\title{
Motivos para estudiar en la vejez: Estructura factorial de Educational Participation Scale (EPS A-form)
}

\author{
Susana Menéndez Álvarez-Dardet \\ Universidad de Huelva \\ mail: menendezQuhu.es \\ ORCID: https://orcid.org/0000-0003-2198-8006
}

\author{
Javier Pérez-Padilla \\ Universidad de Jaén \\ mail: jppadillaujaen.es \\ ORCID: https://orcid.org/0000-0003-2529-348X
}

\author{
Carmen Santín Vilariño \\ Facultad de Educación, Psicología y Ciencias del Deporte. \\ mail: santindadpsi.uhu.es \\ ORCID: https://orcid.org/0000-0002-1387-9275
}

\author{
Enrique Torres Álvarez \\ Universidad de Huelva \\ mail: etorresवuhu.es \\ ORCID: https://orcid.org/0000-0001-8531-7696
}

\begin{abstract}
RESUMEN
Los Programas Universitarios para Mayores (PUM) son un buen recurso para favorecer la educación a lo largo de la vida y, así, dar respuesta a las principales recomendaciones sociopolíticas para fomentar el envejecimiento activo. Una de las dimensiones clave para el estudio de estos programas es el tipo de razones por las que los mayores se implican en ellos, aspecto ampliamente evaluado a nivel internacional mediante la escala EPS A-form. En este trabajo se analiza la estructura factorial de la versión española de esta prueba, utilizando procedimientos estadísticos coherentes con las directrices actuales para este tipo de análisis, en una muestra de estudiantes del Aula de la Experiencia de la Universidad de Huelva (España). Los resultados indican que la versión española de EPS A-form tiene la misma estructura en cuatro subescalas que la original, con índices de consistencia interna entre .83 y .96. Las motivaciones más destacadas fueron las de tipo cognitivo, aunque más de la mitad de los estudiantes participaban en el PUM por más de una razón. Esta escala es una herramienta eficaz de cara al estudio de las motivaciones para estudiar en la vejez, y puede ser útil tanto en la investigación como en el diseño de PUM en España.
\end{abstract}

Palabras Clave: educación a lo largo de la vida; motivación; evaluación; análisis factorial.

\section{Reasons to learn in older age: Factorial structure of Educational Participation Scale (EPS A-form)}

\begin{abstract}
University Programs for Older People (UPO) are a valuable resource to promote lifelong learning and thus respond to the main sociopolitical recommendations for promoting active ageing. One of the key dimensions for the study of these programs is the type of motivations for the older people to be involved in them, a topic widely evaluated at international level using EPS A-form. In this paper the factor structure of the Spanish version of this scale is examined, using statistical procedures consistent with current guidelines for this type of analysis, in a sample of students from the Aula de la Experiencia, the UPO of the University of Huelva (Spain). Results obtained show that the Spanish version of EPS A-form has a structure in four subscales similar to the original, with internal consistency indices between .83 and .96 . The most outstanding motivations were the cognitive ones, although more than a half of the students participated in the program for more than one reason. This scale is an effective tool to research motivations for studying in old age, and can be useful both in research and in the design of University Programs for Older People in Spain.
\end{abstract}

Keywords: lifelong learning; motivation; assessment; factorial analysis.

ISSN: 0210-2773

DOI: https://doi.org/10.17811/rifie.50.4.2021.815-824 


\section{Introducción}

La educación a lo largo de la vida es uno de los enfoques que actualmente suscita más consenso en el ámbito socioeducativo. Desde esta perspectiva se entiende que los procesos de aprendizaje y educación son una realidad posible y deseable durante el ciclo vital para que, a lo largo de toda su vida, las personas puedan adaptarse a los continuos cambios a los que se enfrentan en las sociedades desarrolladas. Se trata por tanto de una forma dinámica de entender el proceso educativo, que rompe las fronteras tradicionales de edad y espacio: la educación y el aprendizaje no tienen por qué circunscribirse a los 10-15 primeros años de la vida o a la escuela, sino que cualquier edad es apropiada para aprender y cualquier espacio puede ser educativo (Boulton-Lewis, 2010; Jamieson, 2007; Kaplan, 2016). En el caso de la vejez, existe una muy importante evidencia empírica sobre los beneficios que tiene la implicación en actividades educativas para la calidad de vida en esta etapa (Jenkins, 2011; Jenkins y Mostafa, 2015, 2017; Merriam y Kee, 2014; Schmidt-Hertha et al., 2019; Vilaplana, 2010; Villar y Celdrán, 2013).

El convencimiento generalizado sobre la importancia de la educación en la tercera edad encuentra eco en las recomendaciones sociopolíticas internacionales para la promoción del envejecimiento activo, un enfoque positivo y constructivo de la vejez que, en la actualidad, fundamenta las actuaciones en relación con los mayores y el envejecimiento en países desarrollados. En el marco de la Segunda Asamblea Mundial de las Naciones Unidas sobre el Envejecimiento celebrada en Madrid en 2002, la Organización Mundial de la Salud (OMS) definió el envejecimiento activo como el proceso por el que se optimizan las oportunidades de bienestar físico, social y mental durante toda la vida, con el objetivo de ampliar la esperanza de vida saludable, la productividad y la calidad de vida en la vejez (OMS, 2002: 79). En esta formulación inicial la OMS ofrecía diversas recomendaciones para promover el envejecimiento activo organizadas en tres ejes centrales: Salud, Participación y Seguridad; dentro de las actuaciones para el fomento de la Participación, se destacaban las políticas destinadas a Proporcionar oportunidades de educación y aprendizaje durante el ciclo vital (OMS, 2002: 101). Una década más tarde, los expertos reunidos en Brasil por el International Longevity Centre (ILC) revisaron el concepto de envejecimiento activo, sus determinantes, los datos sobre envejecimiento poblacional, y los nuevos retos que éstos plantean. A partir de estos análisis, se establecieron nuevas recomendaciones para fomentar el envejecimiento activo organizadas en cuatro componentes fundamentales: Salud, Aprendizaje permanente, Participación y Seguridad. Por tanto, la educación a lo largo de la vida ha ido ganando importancia como determinante del buen envejecer y se propone, actualmente, no como una recomendación más sino como uno de sus cuatro ejes centrales (ILC, 2015: 46-47).

La oferta desde la universidad de programas formativos específicamente dirigidos a personas mayores es un interesante recurso para dar respuesta a las directrices anteriores (Formosa, 2019; Valle, 2014; Vilaplana, 2010; Villar y Celdrán, 2012). Los Programas Universitarios para Mayores (PUM desde ahora) surgieron en España en 1993 y, desde entonces, se han extendido progresivamente hasta ser en la actualidad una realidad consolidada en la mayoría de las universidades: de acuerdo con los datos de la Asociación Estatal de Programas Universitarios para Mayores (AEPUM), en el curso 2018-2019 estos programas se ofertaban en 48 centros universitarios con un total de 63.173 estudiantes matriculados. Con las lógicas diferencias entre unas universidades y otras, los objetivos de los PUM suelen ser facilitar el acceso de los mayores a la educación y la cultura, promo- viendo su calidad de vida y su participación social y comunitaria (Alfageme, 2007; Orte et al., 2004; Valle, 2014; Vilaplana, 2010). El formato más habitual en nuestro país es el denominado modelo francés, que consiste en un conjunto multidisciplinar de asignaturas, organizadas y programadas como clases durante el año académico, a cargo de docentes de la propia universidad, y desarrolladas en las dependencias de la institución.

Los PUM han atraído el interés de la investigación desarrollada desde diversas disciplinas, sobre todo la Psicología y las Ciencias de la Educación. Uno de los aspectos más analizados han sido las peculiaridades de los estudiantes mayores y su experiencia como aprendices y, más en concreto, su motivación para acercarse a la universidad. De manera consistente, los estudios realizados al respecto ponen de manifiesto que los mayores se implican en PUM fundamentalmente por el proceso de aprender (mantener la actividad intelectual, satisfacer la curiosidad, aprender por placer...) más que por los productos del aprendizaje (obtener un título, distraerse, superar situaciones desagradables...), es decir, la actividad educativa en la vejez parece funcionar (a diferencia de lo que suele suceder a otras edades) como un fin más que como un medio (Alfageme, 2007; Findsen y Formosa, 2016; Lucas-Molina et al., 2015; Pérez-Fuentes et al., 2009; Montoro et al., 2007; Mulenga y Liang, 2008; Valle, 2014; Villar, 2006; Villar et al., 2010). No obstante, y como destacan algunos autores (Findsen y Formosa, 2016; Villar y Celdrán, 2012; Villar et al., 2010), aunque la implicación en PUM y, en general, la educación en la vejez obedece muy fundamentalmente a motivos cognitivo-intelectuales, no se debe infravalorar el papel de otro tipo de motivaciones. De hecho, y a pesar de la relevancia de las razones relacionadas con el aprendizaje y la actividad intelectual, los mayores suelen acercarse a las aulas universitarias por más de una razón (Chen y Wang, 2016; Montoro et al., 2007; Valle, 2014; Villar, 2006; Villar et al., 2010). Así, las motivaciones de tipo social (conocer personas de similar edad y con inquietudes parecidas, compartir experiencias interesantes con los amigos...) suelen ser destacadas como la segunda razón principal para participar en PUM (Alfageme, 2007; Findsen y Formosa, 2016; Montoro et al., 2007; Mulenga y Liang, 2008; Valle, 2014; Villar, 2006; Villar et al., 2010; Vives et al., 2015). Asimismo, y aunque las motivaciones de tipo expresivo (aprender por el placer de aprender) son las más destacadas, los PUM también funcionan para los mayores como una vía para adquirir nuevos conocimientos y aplicarlos activamente en la vida cotidiana, es decir, las motivaciones instrumentales son también importantes (Findsen y Formosa, 2016; Valle, 2014; Villar y Celdrán, 2012; Villar et al., 2010).

Resulta por tanto relevante conocer cuáles son las razones por las que los mayores se implican en programas universitarios, y para ello es importante contar con instrumentos de evaluación. De hecho, en el ámbito de la investigación más general sobre la educación en la vejez se viene destacando la importancia de emplear procedimientos cuantitativos y utilizar escalas adaptadas a esta población (Jenkins y Mostafa, 2015; Schmidt-Hertha et al., 2019). En el caso de las motivaciones para participar en actividades educativas formales más allá de la adolescencia, uno de los instrumentos más utilizados a nivel internacional es Education Participation Scale Alternative-form (EPS A-form). Esta escala fue diseñada por Boshier (1991) para evaluar las razones por las que los adultos se implican en programas formativos. La versión original de este autor estaba compuesta por 42 razones agrupadas en siete factores (Mejora de la comunicación; Contacto social; Preparación educativa; Promoción profesional; Cercanía familiar; Estimulación social; Interés cognitivo) con una escala Likert de respuesta desde 1 (Nada) hasta 4 (Mucho). En 2004 Kim y Merrian propusieron una adaptación de la prueba específica para 
personas mayores, eliminando tres subescalas que no tenían sentido en este colectivo de estudiantes, dos por su condición de jubilados (Preparación educativa; Promoción profesional) y una por tener completados estudios básicos (Mejora de la comunicación, que incluye ítems sobre estrategias básicas de lecto-escritura). La versión para estudiantes mayores de Kim y Merrian (2004) se compone de 24 ítems organizados en cuatro subescalas (Contacto social; Cercanía familiar; Estimulación social; Interés cognitivo), con índices de consistencia interna que oscilaron entre $\alpha=.68$ y $\alpha=.92$ en el estudio citado, y ha sido ampliamente utilizada para analizar las motivaciones para estudiar en la universidad en la vejez (Chen y Wang, 2016; Mulenga y Liang, 2008; Xiong y Zuo, 2019). De manera consistente, en estos estudios la subescala con puntuaciones más altas es Interés cognitivo, seguida de Estimulación social, a mucha distancia de ambas Cercanía familiar y, finalmente, Contacto social.

La versión para estudiantes mayores de EPS A-form de Kim y Merrian (2004) fue adaptada a nuestro idioma (mediante la traducción-retrotraducción por parte de dos expertos bilingües) por Villar (2006; Villar et al., 2010) en un estudio sobre los PUM de cuatro universidades españolas. En esta investigación se examinó la estructura factorial de la escala, y se obtuvieron cuatro factores similares a la versión original (la denominación propuesta fue motivos Cognitivos, Sociales, Familiares y Escapismo), con índices de consistencia interna entre $\alpha=.67$ y $\alpha=.91$. En la misma línea que todas las investigaciones que han utilizado EPS A-form, en el estudio español de Villar (2006, Villar et al., 2010) las puntuaciones más elevadas fueron las de las subescalas de motivos Cognitivos y Sociales, y los resultados más bajos fueron los obtenidos para motivos Familiares y Escapismo.

EPS A-form es por tanto un instrumento interesante para evaluar las razones para seguir estudiando en la vejez, con una trayectoria previa importante a nivel internacional, y del que existe una versión en español que ofrece resultados similares a los obtenidos con la escala original. No obstante, algunas decisiones adoptadas en el análisis factorial desarrollado por Villar (2006) (especialmente el uso de Análisis de Componentes Principales como método de estimación, y trabajar con la matriz de correlaciones Pearson con datos ordinales y no continuos como es el caso de las escalas Likert de respuesta) han sido cuestionadas en los últimos años por los especialistas en análisis de datos en ciencias sociales (véase por ejemplo Ferrando y Anguiano-Carrasco, 2010; Ferrando y Lorenzo-Seva, 2014; Hair et al., 2014; Tabachnick y Fidell, 2019). El objetivo de este trabajo es analizar la estructura de la versión española de EPS A-form con estudiantes de un PUM, utilizando procedimientos y criterios estadísticos coherentes con las recomendaciones metodológicas recientes sobre análisis factorial.

\section{Método}

\section{Objetivos}

1) Examinar la estructura factorial de la versión española para personas mayores de EPS A-Form.

2) Analizar la variabilidad de las motivaciones para implicarse en un PUM.

\section{Población y Muestra}

La muestra estaba formada por 301 estudiantes del PUM de la Universidad de Huelva (España). Su edad media eran 65,05 años ( $D T=6,74$, rango 51-86), en concreto un $29,15 \%(n=86)$ tenía hasta 60 años, un $42,03 \%(n=124) 61-69$ años, y un $28,81 \%(n=85) 70$ o más años. El 52,35\% $(n=156)$ eran mujeres y el $47,75 \%(n=142)$ hombres. Un 35,03\% $(n=103)$ tenía estudios previos básicos, un $36,05 \%(n=106)$ estudios secundarios y un $28,91 \%(n=85)$ estudios superiores. La mayoría $(71,33 \%, n=211)$ convivía en pareja, el resto eran viudos $(11,82 \%, n=35)$, separados/divorciados $(9,79 \%, n=29)$ o solteros $(7,09 \%, n=21)$.

\section{Instrumento}

Se utilizó un cuestionario estructurado que, junto a datos sociodemográficos (edad, sexo, nivel educativo previo, y situación de pareja) recogía información sobre las razones para implicarse en el PUM mediante la versión española para personas mayores (Villar, 2006; Villar et al., 2010) de EPS A-form (Kim y Merrian, 2004) (Anexo 1). Como ya se ha señalado, la escala se compone de 24 ítems y ofrece puntuaciones de cuatro subescalas: Motivos Sociales (conocer gente nueva, relacionarse con gente agradable, disfrutar con amigos...), Familiares (ayuda en la comunicación con hijos y nietos, compartir intereses con la familia, estar al día con personas de la familia...), Cognitivos (aprender por el placer de aprender, adquirir conocimiento, satisfacer la curiosidad...), y Escapismo o evitar situaciones indeseables (huir de la soledad, romper con la rutina, no estar inactivo...).

\section{Procedimiento de recogida y análisis de datos}

Se concertó con los responsables del PUM de la Universidad de Huelva (España) un calendario de visitas a las aulas durante el horario de clase para que los estudiantes cumplimentaran el cuestionario que se acaba de describir. En todos los casos estuvieron presentes dos miembros del equipo de investigación para: a) describir los objetivos del estudio, aclarando el carácter anónimo y voluntario de la participación en el mismo; y b) resolver posibles dudas respecto al contenido del cuestionario y/o la forma de responder al mismo. Todos los estudiantes que asistieron a clase los días que se llevó a cabo el trabajo de campo participaron el mismo, completando el cuestionario descrito en el apartado anterior.

Para comprobar la estructura de EPS A-form se computó un Análisis Factorial Restricto (AFR desde ahora) (Ferrando y Anguiano-Carrasco, 2010; Ferrando y Lorenzo-Seva, 2014; Lloret-Segura et al., 2014) con el programa FACTOR-9.2 (Lorenzo-Seva y Ferrando, 2006). Se optó por este procedimiento y este programa porque permiten, respectivamente: a) pre-definir, en base a consideraciones teóricas y a la evidencia empírica previa, el número de dimensiones subyacentes a una escala (los factores) sin especificar los ítems que los componen, de manera que el programa agrupa los que mejor cumplen criterios psicométricos estandarizados para integrar cada dimensión; y b) trabajar con la matriz de correlaciones policóricas, que es la indicada para variables ordinales como las escalas Likert de respuesta (Elosúa y Zumbo, 2008; Ferrando y Lorenzo-Seva, 2014; Lloret-Segura et al., 2014). Antes de computar el AFR se examinó la existencia de casos extremos uni y multivariantes, mediante el examen de los diagramas de caja y bigotes y el cálculo de la distancia de Mahalanobis, respectivamente (Abad et al., 2011; Hair et al., 2014; Pardo et al., 2009, Tabachnick y Fidell, 2019), y se llevó a cabo un análisis métrico de los ítems considerando los siguientes criterios (Abad et al., 2011; Ferrando y Anguiano-Carrasco, 2010; Pardo et al., 2009): asimetría y curtosis dentro de \pm 1 y \pm 2 respectivamente, $r$ ítem-total corregida $>$ 0,30, y descenso de $\alpha$ si se elimina el elemento. De acuerdo con Abad et al. (2011), los dos últimos análisis se hicieron para cada subescala y no para el total de la prueba. Los criterios para establecer la dimensionalidad de la matriz fueron un valor $>0,80$ en el test de Kaiser-Meyer-Olkin (KMO), un resultado significativo en 
la prueba de esfericidad de Barlett, y una determinante cercana a 0 (Hair et al., 2014; Pardo et al., 2009; Tabachnick y Fidell, 2019). Atendiendo a la evidencia (teórica y empírica) previa se solicitó la definición de cuatro factores, utilizando Mínimos Cuadrados no Ponderados como método de estimación y la rotación oblícua Promin, dada la esperable relación entre los factores (Ferrando y Anguiano-Carrasco, 2010; Ferrando y Lorenzo-Seva, 2014; Lloret-Segura et al., 2014). Los criterios para estimar la estructura de la prueba fueron comunalidades $>0,50$, cargas factoriales $>0,30$ en la matriz de saturación, y el valor máximo de cada ítem en cada factor en la matriz de estructura (Ferrando y Anguiano-Carrasco, 2010; Hair et al., 2014; Tabachnick y Fidell, 2019). Para evaluar el ajuste del modelo se tuvieron en cuenta el indicador global de bondad de ajuste GFI, los índices de simplicidad S y LS, y la raíz cuadrada media de los residuos (RMSR); los niveles de referencia fueron GFI $\geq 0,95$, S y LS situados en un percentil cercano a 100, y RMSR $\leq$ 0,08 (Ferrando y Anguiano-Carrasco, 2010; Hair et al., 2014; Tabachnick y Fidell, 2019). La consistencia interna de estimó mediante los índices $\Omega$ de McDonald (para el total de la escala) y $\alpha_{\text {ordinal }}$ (para cada subescala), tomando en ambos casos como criterio un valor > 0,70 (Elosúa y Zumbo, 2008; Ferrando y Anguiano-Carrasco, 2010).

Para transformar las cuatro puntuaciones continuas de las subescalas de EPS A-form en indicadores dicotómicos que reflejaran en qué medida cada tipo de motivación era destacada por cada estudiante del PUM, se ponderaron las cuatro puntuaciones continuas dividiéndolas entre el número de ítems de cada subescala obteniendo así cuatro indicadores ordinales con valores comprendidos dentro del rango de respuesta de la prueba (1-4). Estas nuevas puntuaciones fueron a su vez recodificadas manera dicotómica, considerando que cada tipo de motivación era destacable en cada participante para valores $\geq 3(1=s i)$ y no para valores $<3$ $(0=n o)$. La suma de estos cuatro indicadores dicotómicos ofreció un indicador global sobre el número de motivaciones diferentes de cada estudiante para implicarse en el PUM (rango 0 - 4). Estos análisis fueron realizados con IBM SPSS-23 (IBM, 2015).

\section{Resultados}

No se detectaron extremos univariantes, pero el cálculo de la distancia de Mahalanobis mostró un total de $n=13$ casos extremos multivariantes que fueron excluidos para el resto de los análisis. El examen métrico de los 24 ítems de EPS A-form con los $n=288$ estudiantes restantes se resume en la Tabla 1: los estadísticos de asimetría y curtosis se situaron en valores aceptables $( \pm 1$ $\mathrm{y} \pm 2$, respectivamente), la correlación ítem-total corregida fue $>$ 0,30 , y el $\alpha$ de la subescala descendía si se eliminaba el elemento. Las únicas excepciones fueron los ítems 11, 12 y 24, con valores de asimetría fuera del intervalo \pm 1 pero muy próximos al mismo, y dado que cumplían el resto de los criterios se decidió conservarlos. Por tanto, se retuvieron los 24 ítems para el resto de los análisis.

Tabla 1

Análisis métrico de los ítems de EPS A-form

\begin{tabular}{|c|c|c|c|c|c|c|c|}
\hline Subescala $(\alpha)$ & Ítem & $M$ & $D T$ & Asimetría & Curtosis & $r$ ítem-total corregida & $\alpha$ si se elimina el ítem \\
\hline \multirow[t]{6}{*}{ M. Sociales $(0,92)$} & 1 & 2,28 & 0,82 & 0,38 &,- 027 & 0,76 & 0,90 \\
\hline & 5 & 2,45 & 0,83 & 0,26 & $-0,48$ & 0,83 & 0,89 \\
\hline & 9 & 2,35 & 0,77 & 0,40 & $-0,14$ & 0,79 & 0,90 \\
\hline & 13 & 2,73 & 0,81 & $-0,20$ & $-0,42$ & 0,71 & 0,91 \\
\hline & 17 & 2,53 & 0,96 & 0,01 & $-0,94$ & 0,73 & 0,91 \\
\hline & 21 & 2,42 & 0,84 & 0,25 & $-0,48$ & 0,80 & 0,90 \\
\hline \multirow[t]{6}{*}{ M. Familiares $(0,88)$} & 2 & 2,05 & 0,97 & 0,45 & $-0,92$ & 0,73 & 0,85 \\
\hline & 6 & 2,08 & 1,02 & 0,47 & $-0,96$ & 0,78 & 0,84 \\
\hline & 10 & 2,43 & 0,91 & $-0,01$ & $-0,82$ & 0,65 & 0,86 \\
\hline & 14 & 2,09 & 0,97 & 0,44 & $-0,87$ & 0,72 & 0,85 \\
\hline & 18 & 1,76 & 0,91 & 1,00 & 0,09 & 0,70 & 0,86 \\
\hline & 22 & 2,77 & 0,96 & $-0,35$ & $-0,80$ & 0,54 & 0,87 \\
\hline \multirow[t]{6}{*}{ M. Cognitivos $(0,72)$} & 3 & 3,45 & 0,61 & $-0,75$ & 0,07 & 0,43 & 0,69 \\
\hline & 7 & 3,28 & 0,74 & $-0,65$ & $-0,39$ & 0,47 & 0,68 \\
\hline & 11 & 3,41 & 0,70 & $-1,18$ & 1,64 & 0,39 & 0,70 \\
\hline & 15 & 3,21 & 0,78 & $-0,82$ & 0,34 & 0,45 & 0,68 \\
\hline & 19 & 2,96 & 0,86 & $-0,52$ & $-0,37$ & 0,53 & 0,66 \\
\hline & 23 & 2,72 & 0,98 & $-0,30$ & 0,90 & 0,48 & 0,68 \\
\hline \multirow[t]{6}{*}{ Escapismo $(0,85)$} & 4 & 1,84 & 1,01 & 0,94 & $-0,31$ & 0,71 & 0,82 \\
\hline & 8 & 1,94 & 1,03 & 0,76 & $-0,65$ & 0,78 & 0,80 \\
\hline & 12 & 1,79 & 0,99 & 1,04 & $-0,10$ & 0,73 & 0,81 \\
\hline & 16 & 2,99 & 0,96 & $-0,65$ & $-0,54$ & 0,50 & 0,84 \\
\hline & 20 & 2,58 & 0,99 & $-0,06$ & $-1,04$ & 0,63 & 0,83 \\
\hline & 24 & 1,52 & 0,93 & 1,65 & 1,44 & 0,50 & 0,84 \\
\hline
\end{tabular}

El examen de la dimensionalidad de la matriz de datos también ofreció resultados satisfactorios: $\mathrm{KMO}=0,87$, prueba de esfericidad de Bartlett significativa $\left(\chi^{2}=1272,5, p<0,001\right)$, y determinante $=0,024$. De acuerdo con las evidencias previas del constructo y de la escala, se computó un AFR solicitando cuatro factores. Los resultados obtenidos (Tabla 2) confirman la estructura original de EPS A-form: en todos los ítems la comunalidad fue $>0.50$, y tanto las cargas factoriales de la matriz de saturación, como los valores máximos de la matriz de estructura, indicaron que los ítems se agrupaban en cuatro factores coherentes con las 
cuatro dimensiones evaluadas por EPS A-form: motivos Cogniti$\operatorname{vos}(\mathrm{F} 1$, ítems 3, 7, 11, 15, 19 y 23), Sociales (F2, ítems 1, 5, 9, 13, 17 y 21), Familiares (F3, ítems 2, 6, 10, 14, 18 y 22), y Escapismo (F4, ítems 4, 8, 12, 20 y 24). Los indicadores de bondad de ajuste (GFI $=0,99, S=0,958$ y LS = 0,554-ambos en el Percentil 100-, y RMSR
$=0,040$ ) fueron satisfactorios y avalan la adecuación del modelo. En el mismo sentido apuntan los resultados sobre la consistencia interna de la escala en este estudio $\left(\Omega=0,93 ; \alpha_{\text {ordinal }}\right.$ de cada subescala: $\mathrm{MC}=0,83, \mathrm{MS}=0,96, \mathrm{MF}=0,93, \mathrm{E}=0,94)$.

Tabla 2

Resultados del AFR

\begin{tabular}{|c|c|c|c|c|c|c|c|c|c|}
\hline \multirow{2}{*}{ Ítem } & \multirow{2}{*}{ Comunalidad } & \multicolumn{4}{|c|}{ Matriz de saturación* } & \multicolumn{4}{|c|}{ Matriz de estructura** } \\
\hline & & F1 & F2 & F3 & $\mathbf{F} 4$ & F1 & F2 & F3 & F4 \\
\hline 1 & 0,873 & & 0,75 & & & 0,19 & 0,84 & 0,63 & 0,63 \\
\hline 2 & 0,930 & & & 0,83 & & 0,12 & 0,58 & 0,83 & 0,56 \\
\hline 3 & 0,693 & 0,64 & & & & 0,62 & 0,07 & 0,08 & $-0,01$ \\
\hline 4 & 0,882 & & & & 0,76 & $-0,05$ & 0,63 & 0,60 & 0,83 \\
\hline 5 & 0,992 & & 1,07 & & & 0,21 & 0,93 & 0,59 & 0,57 \\
\hline 6 & 1,001 & & & 0,91 & & 0,18 & 0,61 & 0,88 & 0,56 \\
\hline 7 & 0,599 & 0,61 & & & & 0,61 & 0,10 & 0,20 & 0,09 \\
\hline 8 & 0,948 & & & & 1,06 & 0,01 & 0,55 & 0,51 & 0,92 \\
\hline 9 & 0,983 & & 0,93 & & & 0,17 & 0,88 & 0,62 & 0,58 \\
\hline 10 & 0,823 & & & 0,69 & & 0,24 & 0,52 & 0,73 & 0,49 \\
\hline 11 & 0,651 & 0,66 & & & & 0,64 & 0,13 & $-0,02$ & 0,06 \\
\hline 12 & 0,947 & & & & 0,76 & $-0,02$ & 0,57 & 0,61 & 0,83 \\
\hline 13 & 0,840 & & 0,66 & & & 0,28 & 0,77 & 0,56 & 0,60 \\
\hline 14 & 0,817 & & & 0,78 & & 0,15 & 0,54 & 0,80 & 0,59 \\
\hline 15 & 0,762 & 0,58 & & & & 0,61 & 0,31 & 0,23 & 0,29 \\
\hline 16 & 0,746 & 0,34 & & & 0,74 & 0,40 & 0,39 & 0,35 & 0,63 \\
\hline 17 & 0,875 & & 0,48 & & 0,30 & 0,21 & 0,78 & 0,67 & 0,72 \\
\hline 18 & 0,862 & & & 0,81 & & 0,26 & 0,60 & 0,82 & 0,53 \\
\hline 19 & 0,723 & 0,52 & & 0,31 & & 0,60 & 0,40 & 0,46 & 0,31 \\
\hline 20 & 0,828 & & & & 0,71 & 0,33 & 0,53 & 0,50 & 0,73 \\
\hline 21 & 0,879 & & 0,87 & & & 0,25 & 0,85 & 0,59 & 0,56 \\
\hline 22 & 0,758 & 0,45 & & 0,57 & & 0,56 & 0,48 & 0,63 & 0,35 \\
\hline 23 & 0,932 & 0,54 & & 0,43 & & 0,64 & 0,50 & 0,55 & 0,44 \\
\hline 24 & 0,878 & & & & 0,37 & 0,02 & 0,60 & 0,63 & 0,66 \\
\hline
\end{tabular}

*cargas > 0,30; **en negrita el valor más alto de cada ítem en cada factor

La Tabla 3 resume los datos de tendencia central y dispersión de las cuatro subescalas de EPS A-form. En la Tabla 4 se ofrecen los resultados de los análisis de correlación entre las puntuaciones de las cuatro subescalas, así como la comparación entre cada par de ellas. Los contrastes $t$ para muestras pareadas pusieron de manifiesto diferencias estadísticamente significativas entre todas las motivaciones exceptuando las dos menos destacadas (las Familiares y el Escapismo; ver Tablas 3 y 4). Por tanto, las razones más relevantes entre los participantes en este estudio para implicarse en el PUM fueron las Cognitivas, seguidas de las Sociales y, finalmente (y en la misma medida), las Familiares y el Escapismo (ver Tabla 3). No obstante todas las correlaciones fueron positivas y estadísticamente significativas (ver Tabla 4), indicando así que los estudiantes con motivaciones más relevantes en un ámbito también participaban en el programa por los otros tipos de razones.
Para analizar el número de motivos diferentes para participar en el programa, se transformaron las cuatro puntuaciones continuas de EPS en indicadores dicotómicos y se generó un indicador cuantitativo sobre el número de razones diferentes (ver apartado Análisis estadístico). Las motivaciones Cognitivas fueron destacadas por el 91,7\% de los participantes, las Sociales por el 50,2\%, las Familiares por el 37,5\% y el Escapismo por el 30,9\%. En cuanto al número de razones diferentes para estudiar en la vejez, la media fue $2,1(D T=1,26)$ : sólo un $6,6 \%$ de los estudiantes no destacaron ninguno de los cuatro tipos de motivaciones, mientras que el 34,2\% destacó una, y el 59,1\% más de una $(22,3 \%$ dos, $15,9 \%$ tres, y $20,9 \%$ las cuatro). 
Tabla 3

Subescalas de EPS: puntuaciones de tendencia central y de dispersión.

\begin{tabular}{lcccc}
\hline & MS & MF & MC & E \\
\hline Media & 14.87 & 13.18 & 18.97 & 12.77 \\
DT & 4.30 & 4.53 & 3.06 & 4.52 \\
Rango & $6-24$ & $6-24$ & $10-24$ & $6-24$ \\
Mediana & 15 & 12 & 19 & 12 \\
Moda & 12 & 12 & 20 & 10 \\
Percentil 25 & 12 & 10 & 17 & 9 \\
Percentil 50 & 15 & 12 & 19 & 12 \\
Percentil 75 & 18 & 16 & 21 & 15 \\
\hline
\end{tabular}

MS = Motivaciones Sociales; $\mathrm{MF}=$ Motivaciones Familiares $; \mathrm{MC}=$ Motivaciones Cognitivas $; \mathrm{E}=$ Escapismo

Tabla 4

Análisis bivariantes de la relación entre las subescalas de EPS.

\begin{tabular}{lcccccccc}
\hline & \multicolumn{3}{c}{$r$} & \multicolumn{3}{c}{$t$} \\
\hline & 1 & 2 & 3 & 4 & 1 & 2 & 3 & 4 \\
$1 . \mathrm{MS}$ & - & $0,62^{* * * *}$ & $0,39^{* * * *}$ & $0,66^{* * * *}$ & - & $7,65^{* * * *}$ & $17,01^{* * * *}$ & $9,97^{* * * *}$ \\
$2 . \mathrm{MF}$ & & - & $0,50^{* * * *}$ & $0,57^{* * * *}$ & & - & $25,15^{* * * *}$ & 1,70 \\
$3 . \mathrm{MC}$ & & & - & $0,32^{* * * *}$ & & & - & $23,54^{* * * *}$ \\
$4 . \mathrm{E}$ & & & & - & & & - \\
\hline
\end{tabular}

MS = Motivaciones Sociales; $\mathrm{MF}=$ Motivaciones Familiares; $\mathrm{MC}=$ Motivaciones Cognitivas; $\mathrm{E}=$ Escapismo $* * * * 0<0,001$

\section{Discusión y conclusiones}

El análisis de las motivaciones que promueven la implicación en actividades educativas formales por parte de las personas mayores es uno de los aspectos centrales y más frecuentemente analizados en el marco de la educación a lo largo de la vida. El principal objetivo de este estudio fue explorar la estructura y los resultados de EPS A-form, un instrumento muy utilizado para evaluar las razones para estudiar más allá de la adolescencia, con estudiantes procedentes de un PUM. Los resultados obtenidos indican que la versión española para estudiantes mayores de esta prueba (Villar, 2006; Villar et al., 2010) es una herramienta adecuada y útil para el estudio de las motivaciones que fomentan la participación de las personas mayores en contextos educativos formales.

Por un lado, en este trabajo se ha examinado la estructura de la escala siguiendo las recomendaciones recientes sobre análisis factorial (Ferrando y Anguiano-Carrasco, 2010; Ferrando y Lorenzo-Seva, 2014; Hair et al., 2014; Tabachnick y Fidell, 2019). Los resultados muestran la misma estructura en cuatro factores que la propuesta original (Kim y Merrian, 2004) y la española (Villar, 2006; Villar et al., 2010), y tanto los indicadores de ajuste del modelo como los índices de consistencia interna alcanzaron niveles satisfactorios. Por tanto EPS A-form tiene una estructura psicométricamente sólida, y ofrece información sobre los motivos de los mayores para acercarse a la universidad organizada en cuatro tipos de razones: cognitivo-intelectuales, sociales, familiares y escapismo.

Por otra parte, en este estudio se han analizado las diferentes motivaciones de un grupo de mayores para implicarse en un
PUM, y los resultados están en la línea de lo hallado tanto en nuestro país como en investigaciones de ámbito internacional. Así, las motivaciones más destacadas por los estudiantes mayores de este trabajo son las relacionadas con el placer de pensar y aprender, es decir, las de tipo cognitivo-intelectual, resultado que es coherente con los de otras investigaciones que muestran cómo la educación suele ser, en la vejez, un fin en sí mismo más que un medio (Alfageme, 2007; Chen y Wang, 2016; Findsen y Formosa, 2016; Lucas-Molina et al., 2015; Montoro et al., 2007; Mulenga y Liang, 2008; Pérez-Fuentes et al., 2009; Valle, 2014; Villar, 2006; Villar et al., 2010; Xiong y Zuo, 2019). Por otro lado, y de nuevo al igual que en otros trabajos, los resultados de este estudio muestran que las motivaciones sociales (hacer nuevos amigos, relacionarse con otras personas, conocer gente nueva...) también son muy destacadas y destacables (Chen y Wang, 2016; Findsen y Formosa, 2016; Lucas-Molina et al., 2015; Mulenga y Liang, 2008; Valle, 2014; Xiong y Zuo, 2019). Cabe resaltar que las razones familiares (contestar preguntas de los nietos, mantenerse al día con las nuevas generaciones...) fueron poco frecuentes, al igual que la evitación de situaciones desagradables (aburrimiento, soledad...), siendo estos resultados también consistentes con lo hallado en otros trabajos (Chen y Wang, 2016; Kim y Merrian, 2004; Mulenga y Liang, 2008; Villar, 2006; Villar et al., 2010; Xiong y Zuo, 2019). No obstante, las correlaciones significativas encontradas en este estudio entre todos los tipos de motivaciones, así como el hecho de que la mayoría de los participantes se implicaran en el PUM por más de una razón, indican que involucrarse en un proyecto educativo formal, estructurado y de larga duración, es una decisión compleja que tiende a estar motivada por razones diversas y variadas, como 
se ha destacado en distintos trabajos (Chen y Wang, 2016; Montoro et al., 2007; Valle, 2014; Villar, 2006; Villar et al., 2010). Es decir, las motivaciones cognitivo-intelectuales son importantes y centrales pero no las únicas para que los mayores decidan participar en PUM, y para poder estimar esta diversidad es necesario disponer de herramientas de evaluación sensibles a estas diferencias.

Por tanto, este estudio indica que la versión española de EPS A-form (Villar, 2006, Villar et al., 2010), que se facilita en el Anexo final de este artículo, tiene buenas propiedades psicométricas y permite obtener resultados que están en consonancia con la literatura científica sobre las motivaciones para participar en actividades educativas formales en la vejez. No obstante, consideramos que el trabajo que aquí se presenta tiene algunas limitaciones que conviene señalar. Por un lado, la muestra se ha seleccionado en el PUM de una única universidad y mediante un procedimiento no aleatorio; sería recomendable llevar a cabo otras investigaciones en otras universidades y seleccionando aleatoriamente a los participantes. Asimismo, los resultados de los que aquí se ha informado tienen que ver con las razones para participar en PUM, pero existen otros tipos de actividades educativas de carácter formal (aprendizaje de idiomas, programas de alfabetización digital...) con las que sería conveniente trabajar, comprobando si EPS A-form ofrece resultados similares respecto a las motivaciones de los mayores para implicarse en ellas. Finalmente, queda pendiente y sería deseable abordar el análisis de la validez de esta escala, mediante estudios en los que se incluyan otras dimensiones relacionadas con las motivaciones de los mayores para seguir estudiando en la vejez.

En conclusión, y en línea con las recomendaciones sobre la importancia de emplear procedimientos cuantitativos en la investigación sobre el envejecimiento utilizando escalas específicas para el colectivo de mayores (Jenkins y Mostafa, 2015; Schmidt-Hertha et al., 2019), este trabajo muestra que la versión española de EPS A-form (Villar, 2006; Villar et al., 2010) es un instrumento eficaz y recomendable para evaluar las motivaciones que llevan a estudiar en la universidad en la vejez. Esta escala es por tanto una herramienta útil para la investigación sobre la educación a lo largo de la vida en el contexto hispanohablante, pero también para que los responsables de los PUM de España y Latinoamérica evalúen y analicen las razones por las que los estudiantes mayores se acercan a la universidad, pudiendo así adaptar a estas motivaciones tanto la difusión de esta oferta formativa como su contenido, facilitando así que ambas se ajusten a las necesidades de este colectivo.

\section{Referencias}

Abad, F., Olea, J., Ponsoda, V., y García, C. (2011). Medición en Ciencias Sociales y de la Salud. Síntesis.

Alfageme, A. (2007). The clients and functions of Spanish university programmes for older people: A sociological analysis. Ageing \& Society, 27, 343-361. https://doi.org/10.1017/ S0144686X06005733

Boshier, R. W. (1991). Psychometric properties of the alternative form of the Education Participation Scale. Adult Education Quarterly, 41(3), 150-67.

Boulton-Lewis, G. M. (2010). Education and learning for the elderly: Why, how, what. Educational Gerontology, 36, 213-228. https://doi.org/10.1080/03601270903182877

Chen, L. K., \& Wang, S. T. (2016). Seniors' demographic correlates for motivations to enroll in degree-conferring programs in universities. Educational Gerontology, 42(6), 431-442. https://doi.org/10.1080/03601277.2016.1139968
Elosúa, P., y Zumbo, B. D. (2008). Coeficientes de fiabilidad para respuestas categóricas ordenadas. Psicothema, 20(4), 896-901.

Ferrando, P. J., y Anguiano-Carrasco, C. (2010). El análisis factorial como técnica de investigación en psicología. Papeles del Psicólogo, 31(1), 18-33.

Ferrando, P. J., y Lorenzo-Seva, U. (2014). El análisis factorial exploratorio de los ítems: algunas consideraciones adicionales. Anales de Psicología, 30(3), 1170-1175. https://doi.org/10.6018/ analesps.30.3.199991

Findsen, B., \& Formosa, M. (2016). Participation for and barriers to learning. En B. Findsen \& M. Formosa (Eds.), Lifelong learning in later life: A handbook on older adults learning (pp. 117-130). Sense Publishers.

Formosa, M. (2019). Active ageing through lifelong learning: The University of the Third Age. En The University of the Third Age and Active Ageing: European and Asian-Pacific perspectives (pp. 3-18). Cham.

Hair, J., Black, W.C.; Babin, B.J., \& Anderson, R. (2014). Multivariate Data Analysis (7th ed). Pearson.

IBM Corp. Released (2015). IBM SPSS Statistics for Windows. 23.0 version. IBM Corp.

International Longevity Center (2015). Envejecimiento activo: un marco político ante la revolución de la longevidad. Brasil: International Longevity Center. (Recuperado de https://www. easp.es/project/envejecimiento-activo-un-marco-politico-ante-la-revolucion-de-la-longevidad/).

Jamieson, A. (2007). Higher education study in later life: what is the point? Ageing $\mathcal{E}$ Society, 27, 363-384. https://doi. org/10.1017/S0144686X06005745

Jenkins, A. (2011). Participation in learning and wellbeing among older adults. International Journal of Lifelong Education, 30(3), 403-420. https://doi.org/10.1080/02601370.2011.570876

Jenkins, A., \& Mostafa, T. (2015). The effects of learning on wellbeing for older adults in England. Ageing \& Society, 35(10), 2053. https://doi.org/10.1017/S0144686X14000762

Jenkins, A., \& Mostafa, T. (2017). Wellbeing and learning in later life. International Journal of Education and Ageing, 3(1), 9-24.

Kaplan, A. (2016). Lifelong learning: Conclusions from a literature review. International Online Journal of Primary Education, 5(2), 43-50.

Kim, A., \& Merriam, S. B. (2004). Motivations for learning among older adults in a learning in retirement institute. Educational Gerontology, 30, 441-455. https://doi. org/10.1080/03601270490445069

Lloret-Segura, S., Ferreres-Traver, A., Hernández-Baeza, A., y Tomás-Marco, I. (2014). El análisis factorial exploratorio de los ítems: una guía práctica, revisada y actualizada. Anales de Psicología, 30(3), 1151-1169. https://doi.org/10.6018/analesps.30.3.199361

Lorenzo-Seva, U., \& Ferrando, P. J. (2006). FACTOR. A computer program to fit the exploratory factor analysis model. Behavior Research Methods, 38(1), 88-91. https://doi.org/10.3758/ BF03192753

Lucas-Molina, B., Pérez-Albéniz, A., Fonseca, E., y Ortuño, J. (2015). Programas educativos universitarios para mayores: evaluación de su impacto en la autopercepción del apoyo social y la salud mental. Revista Colombiana de Psicología, 24(1), 47-60. https://doi.org/10.15446/rcp.v24n143437

Merriam, S. B., \& Kee, Y. (2014). Promoting community wellbeing: The case for lifelong learning for older adults. Adult Education Quarterly, 64(2), 128-144. https://doi. org/10.1177/0741713613513633

Montoro, J., Pinazo, S., y Tortosa, M. A. (2007). Motivaciones y expectativas de los estudiantes mayores de 55 años en los 
programas universitarios. Revista Española de Geriatría y Gerontología, 42(3), 158-166. https://doi.org/10.1016/S0211$139 \times(07) 73542-4$

Mulenga, D., \& Liang, J.S. (2008). Motivations for older adults' participation in distance education: A study at the National open university of Taiwan. International Journal of Lifelong Education, 27(3), 289-314. https://doi.org/10.1080/02601370802047791

OMS (2002). Envejecimiento activo. Un marco sociopolítico. Revista Española de Geriatría y Gerontología, 37(S2), 74-105.

Orte, C., Ballester, L1., \& Touza, C. (2004). University programs for seniors in Spain: analysis and perspectives. Educational Gerontology, 30, 315-328. https://doi.org/10.1080/03601270490278849

Pardo, A., Ruiz, M.A. y San Martín, R. (2009). Análisis de datos en ciencias sociales y de la salud (vol. I). Síntesis.

Pérez-Fuentes, M. C., Gázquez, J. J., Lucas, F., Ruiz, I., Amate, R., Miras, F., y Yuste, N. (2009). Satisfacción y motivación del alumnado de la Universidad de Mayores. International Journal of Developmental and Educational Psychology, 1(3), 391-398.

Schmidt-Hertha, B., Formosa, M., \& Fragoso, A. (2019). Active ageing, social inclusion and wellbeing: Benefits of learning in later life. European Journal for Research on the Education and Learning of Adults, 10(3), 207-213. https://doi.org/10.3384/ rela.2000-7426.relae19

Tabachnick, B. G., \& Fidell, L. S. (2019). Using multivariate statistics $\left(7^{\text {th }}\right.$ ed.). Pearson Education.

Valle, F. E. (2014). Educación permanente: los programas universitarios para mayores en España como respuesta a una nueva realidad social. Revista de Educación Superior, XLIII(3), 117-138.
Vilaplana, C. (2010). Relación entre los programas universitarios para mayores, la satisfacción durante la jubilación y la calidad de vida. Revista de Investigación Educativa, 28(1), 195-216.

Villar, F. (2006). Evaluación de programas universitarios para mayores: Motivaciones, dificultades y contribuciones a la calidad de vida. Madrid. Estudios de I+D+I, no 50. (Recuperado de www.imsersomayores.csic.es/documentos/documentos/imserso-estudiosidi-50.pdf).

Villar, F., \& Celdrán, M. (2012). Generativity in older age: a challenge for Universities of the third age (U3A). Educational Gerontology, 38, 666-677. https://doi.org/10.1080/03601277.2011.5 95347

Villar, F., \& Celdrán, M. (2013). Learning in later life: Participation in formal, non-formal and informal activities in nationally representative Spanish simple. European Journal of Ageing, 10, 135-144. https://doi.org/10.1007/s10433-012-0257-1

Villar, F., Pinazo, S., Triadó, C., Celdrán, M., \& Solé, C. (2010). Older people's university students in Spain: a comparison of motives and benefits between two models. Ageing $\mathcal{E}$ Society, 30, 1357-1372. https://doi.org/10.1017/S0144686X10000565

Vives, M., Orte, C., y Ballester, Ll. (2015). Efectos de los programas universitarios en personas mayores en su red y apoyo social. El ejemplo de la Universitat Oberta Per a Majors. Pedagogía Social. Revista Interuniversitaria, 25, 299-317. https://doi. org/10.7179/PSRI2015.25.13

Xiong, J., \& Zuo, M. (2019). Older adults' learning motivations in massive open online courses. Educational Gerontology, 45(2), 82-93. https://doi.org/10.1080/03601277.2019.1581444 
Anexo 1 (EPS A-form)

Participo en el Aula de la Experiencia para:

1. Hacer amistades

2. Ayudarme a hablar y a comunicarme con mis hijos o mis nietos

3. Adquirir conocimientos en general

4. Evitar la soledad

5. Conocer gente nueva

6. Contestar preguntas de mis hijos o mis nietos

7. Adquirir conocimiento por mis propios medios

8. Salir del aburrimiento

9. Conocer gente diferente

10. Compartir intereses con las personas que quiero

11. Aprender por el placer de aprender

12. Superar la frustración del día a día

13. Relacionarme con gente agradable

14. Mantenerme al día con algunas personas de mi familia

15. Satisfacer mi curiosidad

16. Hacer algo y no estar inactivo

17. Disfrutar o pasarlo bien con los amigos

18. Prepararme para nuevos cambios familiares

19. Desarrollar mi imaginación

20. Romper con la rutina de la casa o el trabajo

21. Hacer nuevos amigos

22. Mantenerme al día con las nuevas generaciones

23. Conseguir algo significativo de la vida

24. Evitar una relación infeliz

MS (Motivaciones Sociales $)=1+5+9+13+17+21$

MF (Motivaciones Familiares) $=2+6+10+14+18+22$

MC (Motivaciones Cognitivas) $=3+7+11+15+19+23$

$\mathrm{E}($ Escapismo $)=4+8+12+16+20+24$ 
